Proceedings of the 2011 Winter Simulation Conference

S. Jain, R.R. Creasey, J. Himmelspach, K.P. White, and M. Fu, eds.

\title{
A GENERAL MODEL FOR SOFT BODY SIMULATION IN MOTION
}

\author{
Jaruwan Mesit \\ Ratan K. Guha \\ Department of Electrical Engineering and Computer Science \\ University of Central Florida \\ Orlando, FL 32816, USA
}

\begin{abstract}
Soft bodies are the models in which the bodies deform during animated frames depending on the interaction between themselves and environment. This paper presents a parametric general model for soft body simulation in which structure, deformation, and volume controls generate animated deformations restricted by a set of constraints within or without an environment of gravitation. In this model, the soft body shape is controlled by structure control and anamorphosis of the soft body is created by deformation control, while the mass is approximated by volume control. A set of constraints for these controls further restrict the types of deformation of the soft body. By selecting specific methods for structure, deformation, and volume controls with a set of constraints, we demonstrate a variety of appealing fluid-like surfaces and respiration of lungs for validating the usefulness of the general model.
\end{abstract}

\section{INTRODUCTION}

The objective of this paper is to present a general model for deformable objects. The three dimensional (3D) deformable objects are used in a wide range of applications and a variety of research exists for the modeling of soft body. Most of these research efforts emphasize on specific type of soft bodies or stable simulation techniques for small or large deformations. Here, we state only a handful of different types of modeling and simulation. The earliest work of 3D model has focused on specifying the deformation using the geometric-based modeling. For example, Sederberg and Parry (1986) propose a technique to deform solid geometric models by using free-form deformation (FFD), which can deform surface primitives of any planes, quadrics, parametric surface patches, or implicitly defined surfaces. Chadwick, Haumann, and Parent (1989) present layered construction for deformable animated characters to create and animate the computer generated characters. This methodology combines physics based modeling and geometric modeling with the hierarchy of composite deformations to define both local and global transitions of the character's movement. Recent work on 3D models includes the deformation using linear and non-linear elasticity, geometric constraints, facial deformation, etc. For example, Müller and Gross (2004) propose the method for interactive virtual material where the warped stiffness finite element approach for linear elasticity is combined with a strain-state-based plasticity model. This approach presents the replacement of energies by geometric constraints and forces from the current positions and the goal positions. Botsch et al. (2006) present Primo: coupled prisms for intuitive surface modeling where the surface mesh is embedded in a layer of volumetric prisms with non-linear elastic force. Stoiber, Seguier, and Breton (2010) present facial animation retargeting and control based on human appearance space that combines with the parameter-based animation method to offer a precise control on facial configuration and performance-based animation to naturally capture human motion. Thus, various methods have been used to model soft bodies. 


\section{Mesit and Guha}

By examining most of the soft body modeling techniques, we observe that a soft body deformation is generally based on body structure control, anamorphosis control, volume control, constraints, and gravity. Using these facts, we propose a parametric based general formal soft body model utilizing these components. The body structure control preserves the structure of the model whereas anamorphosis control determines the deformation of the model surface. The volume control defines the variability in the volume of the model. Constraints may impose special relations in these three components. The effect of gravity is determined by the gravity component.

For our general model, specific methods are selected to implement the soft body deformation due to the interaction with environment. Other specific methods can also be selected to implement the proposed general model. For the specific methods, the 3D soft body is defined by a triangular mesh of surface points and the volume inside the soft body is maintained by internal pressure. All control forces are applied to each surface point to model the soft body deformation. Instead of considering all points inside the soft body, internal pressure force maintains an approximated volume without explicit internal structure. This approach significantly reduces simulation time required to determine the configuration of the soft body. In order to show that the general soft body model can be applied to various soft body applications, we select specific control methods and implement soft body models with and without constraints.

Remainder of this paper is structured as follows. Section 2 describes a general formal model of rigid and soft bodies. Section 3 discusses implementation of soft body model using specific methods. Sections 4 and 5 present soft body simulation without and with constraint. Section 6 concludes our work and discusses some possible phenomena that can be modeled by our general soft body model.

\section{A GENERAL FORMAL MODEL OF RIGID AND SOFT BODIES IN MOTIONS}

This section formally describes the $3 \mathrm{D}$ soft body motion model. First, the rigid body in motion, $R 3 D$, is defined as:

$$
R 3 D=\langle O, F, V\rangle,
$$

where $O$ is the object defined as $O=\langle P, E, T\rangle$,

$P=\left\{p_{i}|i=0, . ., n-1|\right\}$ is a set of surface points of the rigid body and $\left(x_{i}, y_{i}, z_{i}\right)$ is a coordinate of surface point $p_{i}$,

$E=\left\{p_{i} p_{j} \mid p_{i}, p_{j} \in P\right\}$ is a set of edges that connect $p_{i}$ and $p_{j}$,

$T=\left\{\begin{array}{l}p_{i} p_{j} p_{k} \mid p_{i}, p_{j}, p_{k} \in P \text {, and } \\ \mid p_{i} p_{j}, p_{i} p_{k} \text {, and } p_{j} p_{k} \in E\end{array}\right\}$ is a set of triangles of rigid body surface defined by $p_{i}, p_{j}$, and $p_{k}$,

$F=\left\{\boldsymbol{F}, \boldsymbol{F}_{g}\right\}$ is a set of forces consisting of $\boldsymbol{F}$, an external force, and $\boldsymbol{F}_{g}$, a gravitational force, applied at the center of the rigid body, and,

$V$ is the velocity at the center of the rigid body.

On the other hand, soft body volume changes due to the pressure applied on the surface of the soft body. This change is related to the composition of the soft body, special characteristics of the soft body, or both. Since our rigid body model considers only surface points for the structures of the body, we present a composition and molecules for representing the volume of the soft body. The specific characteristics are defined by a set of constraints depending on the soft body models. We now formally model the $3 \mathrm{D}$ soft body in motion, $S 3 D$, as

$$
S 3 D=\langle O, F, V, N, C\rangle,
$$

where $O$ is for the soft body as defined in the rigid body model, 


\section{Mesit and Guha}

$F=\left\{\boldsymbol{F}_{i} \mid i=0, . ., n-1\right\}$ is a set of forces for each surface point of the soft body,

$V=\left\{\boldsymbol{v}_{i} \mid i=0, . ., n-1\right\}$ is a set of velocities for each surface point of the soft body,

$N$ is a number proportional to the number of molecules inside the soft body to maintain the approximated volume of the soft body, and

$C$ is a set of deformation constraints for various types of the object being modeled for specific applications.

The force at each point is defined as $F_{i}=\left\langle F_{b i}, F_{a i}, F_{v i}, F_{g i}\right\rangle$, where $\boldsymbol{F}_{b i}^{t}$ is the force of the body structure control at surface point $i, F_{a i}^{t}$ is the force of the anamorphosis control at surface point $i, \boldsymbol{F}_{v i}^{t}$ is the force of the volume control at surface point $i$, and $\boldsymbol{F}_{g i}^{t}$ is the force of gravity at surface point $i$.

If the model uses the structure of a triangle mesh in 3D rigid or soft body in motion, we also define $l_{i j}=\left|p_{i} p_{j}\right|$, length of the edge $p_{i} p_{j} \in E$ and $a_{i j k}=\left|p_{i} p_{j} p_{k}\right|$, area of the triangle, $p_{i} p_{j} p_{k} \in T$. $l_{i j}$ and $a_{i j k}$ are constants for rigid body whereas $l_{i j}$ and $a_{i j k}$ change in soft body due to deformation and interaction with environment. In this model, it is assumed that the number of surface points, edges, and triangles remain constant after any deformation.

To obtain a desired level of softness and deformation, we propose a parametric model of different forces. As indicated previously, the composite force, $\boldsymbol{F}_{i}{ }^{t}$, of the surface point $i$ at time $t$ is based on force components. With this assumption, $\boldsymbol{F}_{i}^{t}$ is defined as:

$$
\boldsymbol{F}_{i}^{t}=\alpha \boldsymbol{F}_{b i}^{t}+\beta \boldsymbol{F}_{f i}^{t}+\gamma \boldsymbol{F}_{v i}^{t}+\delta \boldsymbol{F}_{g i}^{t},
$$

Where parameters, $\alpha, \beta, \gamma$, and $\delta$ are parameters of body structure control, anamorphosis control, volume control, and gravitational field, respectively, where $\alpha>0$ and $\beta, \gamma, \delta \geq 0$. Constraints may exist for relations among these components.

The purpose of each parameter is as follows:

- The value of $\alpha>0$ determines impact of body structure control, enabling the model to deform while maintaining a relative configuration among the surface points. If body structure control (structure of the model) is significant for modeling, then the value of $\alpha$ may dominate among all parameter values.

- The value of $\beta \geq 0$ affects deformation of the soft body. If deformation is important for the model, then the value of $\beta$ may dominate among all parameter values.

- The value of $\gamma \geq 0$ influences body volume as well as compression and decompression due to the molecules inside. If the volume of the model is important for the model, then the value of $\gamma$ should be carefully determined.

- The value of $\delta \geq 0$ simulates the soft body with or without the impact of gravitational force. $\delta=0$ is for no gravity.

In this manner, the various force parameters exerted on each soft body surface point can be adjusted to obtain specific types of soft body behaviors. 


\section{Mesit and Guha}

\section{IMPLEMENTATION OF SOFT BODY MODEL USING SPECIFIC METHODS}

In order to implement the general soft body model, we need to select specific control methods for the force at each surface point. For modeling, the mass-spring system, SPH fluidity model, and internal pressure are selected for body structure control, surface anamorphosis control, and volume control, respectively. The environment of this model can be with or without gravity. The detail of each control is described here.

For modeling body structure control, we select a mass spring system. The force, $\boldsymbol{F}_{b i}^{t}$, generated by the mass spring system is determined by two parts, spring force $\boldsymbol{F}_{s i}^{t}$ and damping force $\boldsymbol{F}_{d i}^{t}$. Thus, $\boldsymbol{F}_{b i}^{t}$ is defined as follows (Bourg 2001; Desbrun, Schröder, and Barr 1999):

$$
\boldsymbol{F}_{b i}^{t}=\boldsymbol{F}_{s i}^{t}+\boldsymbol{F}_{d i}^{t},
$$

where $\boldsymbol{F}_{s i}^{t}$ is spring force at surface point $i$ and $\boldsymbol{F}_{d i}^{t}$ is damping force at surface point $i$.

In a mass-spring system, the spring force generated by each spring depends on the spring constant, the spring length, and spring rest position. The equation for cumulative spring force among neighboring surface points is given by:

$$
\boldsymbol{F}_{s i}^{t}=\sum_{\forall j(i, j) \in E} k_{s}\left(l_{i j}^{t}-l_{i j}^{0}\right) \frac{\left(p_{j}^{t}-p_{i}^{t}\right)}{l_{i j}^{t}},
$$

where $\boldsymbol{F}_{s i}^{t}$ is the net internal spring force exerted on surface point $i$ for every surface point $j$ in the neighborhood $E, k_{s}$ is the spring constant of the spring connecting surface points $i$ and $j, p_{i}$ and $p_{j}$ are the positions of surface point $i$ and $j$ respectively, $l_{i j}^{0}$ is the initial length of the spring between surface points $i$ and $j$, and $t$ is current time step.

Damping force works against the velocities of connected surface points in order to slow their relative velocities. The equation of cumulative damping force among a surface point's neighbors is given by:

$$
\boldsymbol{F}_{d i}^{t}=\sum_{\forall j \mid(i, j) \in E} k_{d} h\left(\boldsymbol{v}_{i}^{t}-\boldsymbol{v}_{j}^{t}\right)
$$

where $\boldsymbol{F}_{d i}^{t}$ is the damping force, $k_{d}$ is the damping constant between surface point $i$ and $j, \boldsymbol{v}_{i}$ is the velocity at surface point $i, \boldsymbol{v}_{j}$ is the velocity at surface point $j, t$ is current time step, and $h$ is time elapsed.

In order to model complex surface interactions, forces beyond mass-spring systems are required. For example the fluid-like deformation on the surface of a deformed soft body. Since 3D fluid simulation is computationally expensive, we perform fluid calculation on the mass-spring surface of the soft body only while we generate the volume by internal pressure force which acts on each surface point.

For modeling behavior of fluids, we simulate the surface points as free moving particles interacting with nearby surface points within a radius. We adopt the smoothed particle hydrodynamics (SPH) model developed originally for astrophysics problems and later used in interactive applications of particles based on fluid simulation (Müller, Charypar, and Gross 2003; Müller et al. 2005). In general, SPH is simple compared to other fluid modeling such as FEM or FVM. SPH is an interpolation method that distributes quantities in a local neighborhood of each particle using radial symmetrical smoothing kernels. We utilize poly6, spiky, and viscosity smoothing kernels in Müller, Charypar, and Gross (2003) and Müller et al. (2005) to model fluid density, fluid pressure, and viscosity forces. Thus, during fluid force calculation, fluid density and fluid pressure are computed to generate fluid pressure force and fluid viscosity force as presented as follows. 


\section{Mesit and Guha}

In accordance with the model developed in Müller, Charypar, and Gross (2003) and Müller et al. (2005), fluid density is given by:

$$
\rho_{i}^{t}=\sum_{j} m_{j} W_{p o l y 6}\left(l_{i j}^{t}, h_{w}\right), \forall j \text { such that } l_{i j}^{t} \leq h_{w} \quad,
$$

where $\rho_{i}^{t}$ is the density at surface point $i$ at time $t, m_{j}$ is the mass at surface point $j$, and $h_{w}$ is the core radius of $\mathrm{SPH}$.

Next, fluid pressure is generated from fluid density as:

$$
L_{i}^{t}=k\left(\rho_{i}^{t}-\rho^{0}\right)
$$

where $L_{i}^{t}$ is the fluid or liquid pressure at surface point $i$ at time $t, k$ is the gas constant, $\rho_{i}^{t}$ is the density at surface point $i$ at time $t$, and $\rho^{0}$ is the initial density.

Fluid pressure force at the soft body surface point $i, \boldsymbol{F}_{f p i}^{t}$, is computed as:

$$
\boldsymbol{F}_{f p i}^{t}=-\sum_{j} m_{j} \frac{L_{i}^{t}-L_{j}^{t}}{2 \rho_{j}^{t}} \nabla W_{s p i k y}\left(l_{i j}^{t}, h_{w}\right), \forall j \text { such that } l_{i j}^{t} \leq h_{w},
$$

where $m_{j}$ is the mass at surface point $j, L_{i}^{t}$ and $L_{j}^{t}$ are fluid or liquid pressure values at surface points $i$ and $j$ respectively at time $t, \rho_{j}^{t}$ is the density at surface point $j$ at time $t$, and $h_{w}$ is the core radius of $\mathrm{SPH}$.

Finally the fluid viscosity force at the soft body surface point $i, \boldsymbol{F}_{f v i}^{t}$, is generated by:

$$
\boldsymbol{F}_{f v i}^{t}=\mu \sum_{j} m_{j} \frac{\boldsymbol{v}_{j}^{t}-\boldsymbol{v}_{i}^{t}}{\rho_{j}^{t}} \nabla^{2} W_{v i s c o s i t y}\left(l_{i j}^{t}, h_{w}\right), \forall j \text { such that } l_{i j}^{t} \leq h_{w},
$$

where $\mu$ is the viscosity of fluid, $m_{j}$ is the mass at surface point $j, \boldsymbol{v}_{i}^{t}$ and $\boldsymbol{v}_{j}^{t}$ are the velocities at surface points $i$ and $j$ respectively at time $t, \quad \rho_{j}^{t}$ is the density at surface point $j$ at time $t$, and $h_{w}$ is the core radius of $\mathrm{SPH}$.

The anamorphosis control generated by fluid force, $\boldsymbol{F}_{a i}^{\boldsymbol{t}}$, is the combination of two different forces: (1) fluid pressure force, $\boldsymbol{F}_{f p i}^{t}$, and (2) fluid viscosity force, $\boldsymbol{F}_{f v i}^{t}$. Hence, we define the fluid force as follows:

$$
F_{a i}^{t}=F_{f p i}^{t}+F_{f v i}^{t}
$$

where $\boldsymbol{F}_{f p i}^{t}$ is fluid pressure force at surface point $i$ and $\boldsymbol{F}_{f v i}^{t}$ is fluid viscosity force at surface point $i$.

In order to model volume of the soft body an internal pressure force must push the surface points outward. This volume is created by pressure force generated by the molecules within the soft body. Without volume, the soft body may become flat, much like fabric or cloth after colliding with the environment.

We are proposing a model for maintaining the volume of the soft body by internal pressure force. This model is conceptually similar to the idea presented in Matyka and Ollila (2003). The internal pressure force is defined as: 


$$
\boldsymbol{F}_{v i}^{t}=\sum_{\forall j \notin(i, j, k) \in E} a_{i j k} \hat{n} \frac{1}{B} N
$$

where $\boldsymbol{F}_{v i}^{t}$ is the net pressure force in bounding box volume of soft body experienced at surface point $i$ at time $t, a_{i j k}$ is the surface area of the face connecting surface point $i$ to all surface point pairs $(i, k)$ in the neighborhood of $i, \hat{n}$ is a normal vector to the surface where the pressure force is acting, $B$ is the volume of the soft body bounding box at current frame, and $N$ is proportional to the number of molecules inside the soft body to maintain the approximated volume of the soft body.

In the real-world gravity affects all objects, thus the simulation model must account for it. The force of gravity experienced by an object on earth is equal to weight of the object experiencing the gravitational pull. Gravitational force at a point on the soft body surface is computed by:

$$
\boldsymbol{F}_{g i}^{t}=m_{i} \boldsymbol{g}
$$

where $\boldsymbol{F}_{g i}^{t}$ is gravity force at time $t$ acting on surface point $i$ of mass, $m_{i}$, and $\boldsymbol{g}$ is acceleration due to Earth's gravity.

When computing the sum of all individual forces on a soft body, the forces must be combined in this step for each surface point. The combination of all forces is based on proportional selection of particular forces suitable for specific applications.

Utilizing the specific model, we demonstrate two simulations: one with no constraint and the other with a constraint.

\section{SOFT BODY SIMULATION WITHOUT CONSTRAINT}

In the first demonstration, a soft body is dropped into a rigid-body wine glass. The simulation has six different combinations of parameters $\alpha, \beta, \gamma$, and $\delta$ which are body structure control, anamorphosis control, volume control, and gravitational field, respectively, where $\alpha>0$, and $\beta, \gamma, \delta \geq 0$. Since the selected mass-spring system for body structure control enables the model to deform while maintaining a relative configuration among the surface points without loss of generality, the body structure control is set to one in both scenarios $(\alpha=1)$.

The soft body illustrates the main effects of anamorphosis control from fluid force and volume control from internal pressure force. To demonstrate fluid-like behavior, the fluid force is set to $\beta=0,1,2$. The internal pressure force is adjusted and based on the shape and the purpose of the model. For the shape of balloon-like soft body in first scenario, the internal pressure force is set to $\gamma=0,1,5$, which provides more volume.

We have experimented with six sets of parameter values as shown in Table 1 to demonstrate a variety of soft body behaviors. The visual results of the model depend on the model structures such as the number of surface points and connection of those surface points and are shown in Figures 1-6, each with six animated frames captured at $100^{\text {th }}, 1000^{\text {th }}, 2000^{\text {th }}, 3000^{\text {th }}, 4000^{\text {th }}$, and $6000^{\text {th }}$. The soft body has 1,986 surface points, 3,968 faces, and 5,952 springs. Experiment A shows that a zero fluid parameter value results in a smooth surface of the soft body (see Figure 1). Experiments B and C illustrate variable different fluid parameters that cause different levels of undulating surface waves, and the parameter of fluid force becomes the dominant factor on the soft body as shown in Figures 2 and 3. Experiments D and E have high internal pressure value and behave like bubbles or rubber balls as shown in Figures 4 and 5. Both show that internal pressure force becomes the dominant factor on the soft body. Thus, the fluid force parameter does not affect visual result in Figure 5. Finally, experiment $F$ has a zero internal pressure, which results in a soft body without volume much like fabric or cloth as shown in Figure 6. Overall, the experiment demonstrates the effectiveness of the proposed method to simulate a variety of fluid-like surfaces. 


\section{Mesit and Guha}

Table 1: Experiment parameters for fluid-like soft body deformation experiments

\begin{tabular}{|c|c|c|c|c|c|}
\hline Experiment & $\alpha$ & $\beta$ & $\gamma$ & $\delta$ & FPS \\
\hline A & 1 & 0 & 1 & 1 & $>20$ \\
\hline B & 1 & 1 & 1 & 1 & $>20$ \\
\hline C & 1 & 2 & 1 & 1 & $>20$ \\
\hline D & 1 & 0 & 5 & 1 & $>20$ \\
\hline E & 1 & 2 & 5 & 1 & $>20$ \\
\hline F & 1 & 1 & 0 & 1 & $>20$ \\
\hline
\end{tabular}
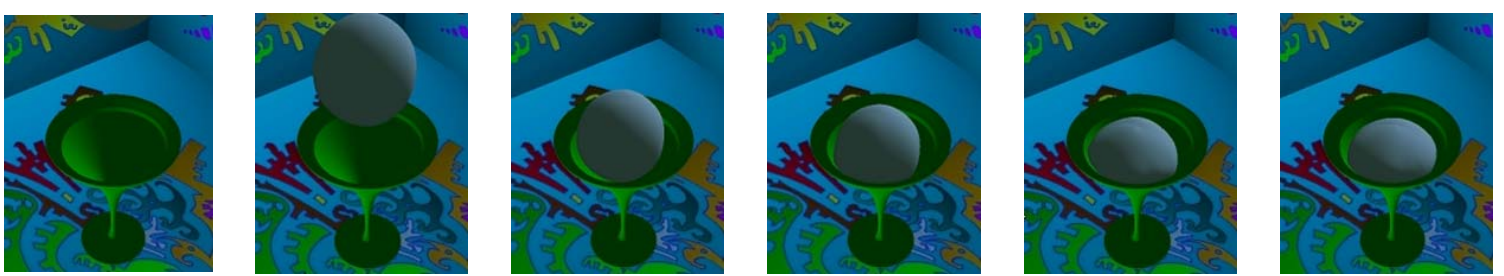

Figure 1: The visual result of soft body model of experiment A, with parameters $\alpha=1, \beta=0, \gamma=1$, and $\delta=1$. The result shows that these parameters generate the smooth surface of the soft body.
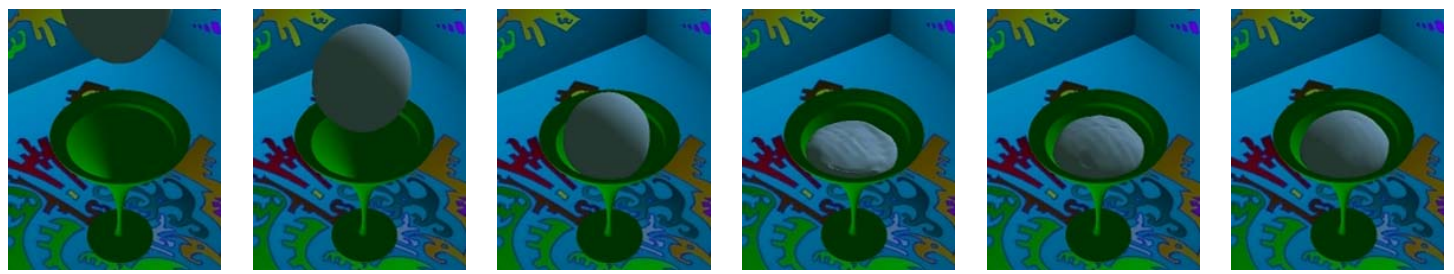

Figure 2: The visual result of soft body model of experiment $\mathrm{B}$, with parameters $\alpha=1, \beta=1, \gamma=1$, and $\delta=1$. The result shows that these parameters generate the undulating surface waves.
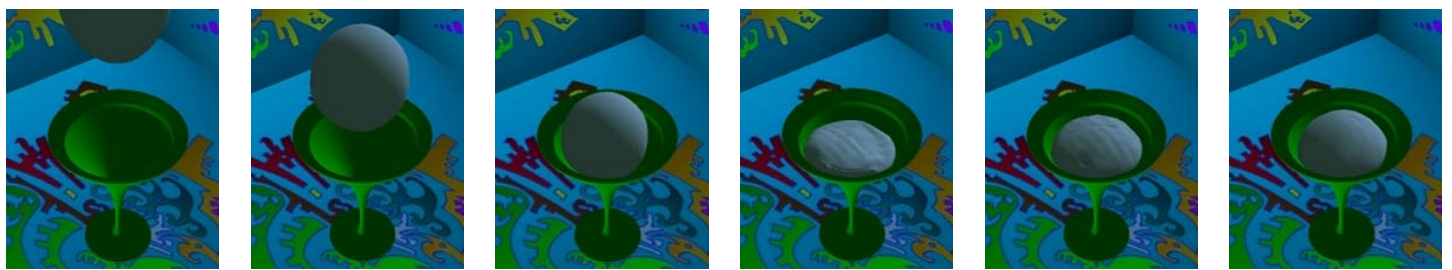

Figure 3: The visual result of soft body model of experiment $\mathrm{C}$, with parameters $\alpha=1, \beta=2, \gamma=1$, and $\delta=1$ have been set. The result shows that these parameters generate more undulating surface waves compared to experiment $\mathrm{B}$.
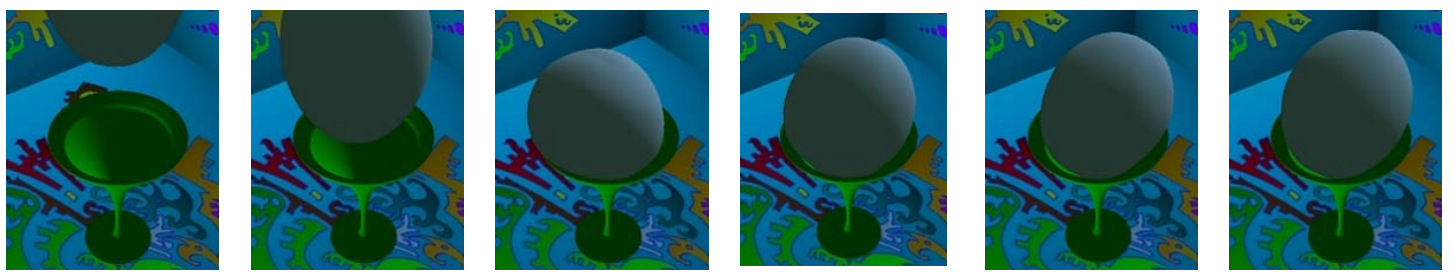

Figure 4: The visual result of soft body model of experiment $\mathrm{D}$, with parameters $\alpha=1, \beta=0, \gamma=5$, and $\delta=1$. The result shows that these parameters generate bubbles or rubber balls. 

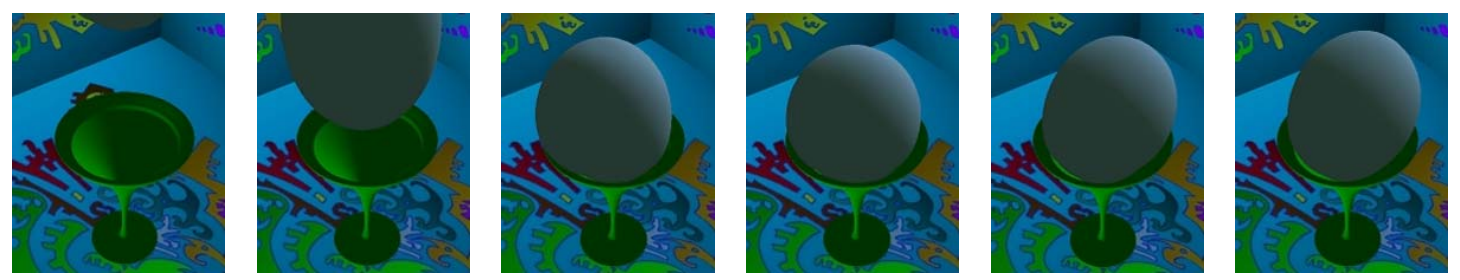

Figure 5: The visual result of soft body model of experiment $\mathrm{E}$, with parameters $\alpha=1, \beta=0, \gamma=2$, and $\delta=1$ have been set. The result shows that these parameters generate bubbles or rubber balls. Since internal pressure force becomes the dominant factor on the soft body, the fluid force parameter does not affect visual result in this experiment.
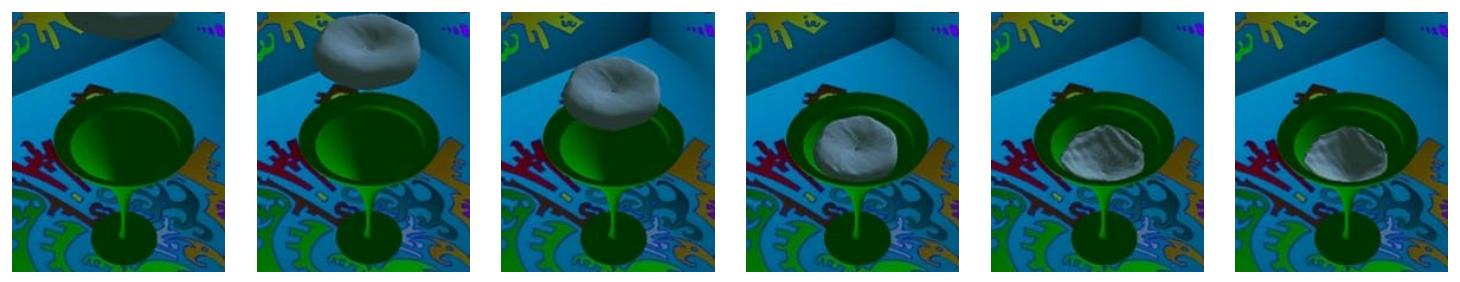

Figure 6 : The visual result of soft body model of experiment $\mathrm{F}$, with parameters $\alpha=1, \beta=1, \gamma=0$, and $\delta=1$ have been set. The result shows that these parameters generate the soft body without volume much like fabric or cloth.

\section{SOFT BODY SIMULATION WITH A CONSTRAINT}

In this section we present the basic functions of the lungs and respiration. A brief explanation of respiration and pressure volume relation of the lungs are described here.

Lung volume or lung capacity is related to the state of the respiratory cycle. During normal breathing, the average of total lung capacity is about 6 liters of air for human male and 4.7 liters of air for a human female (Palsson, Hubbell, and Plonsey 2003). A single inhalation and exhalation involves the movement of thoracic muscles including the muscles of the abdominal region, the diaphragm, and the intercostal muscles. The movement of diaphragm and chest wall form a mechanical pump that drive air flow into and out of the lungs (Cotes 1993, Harris 2005, Santhanam 2003). The change of pressure and volume is known as the Pressure-Volume (P-V) relation. The relation of P-V is shown in Figure 7. This relationship indicates changes in pulmonary mechanics and reflects the respiratory parameters such as lung tissue properties (Santhanam 2003).

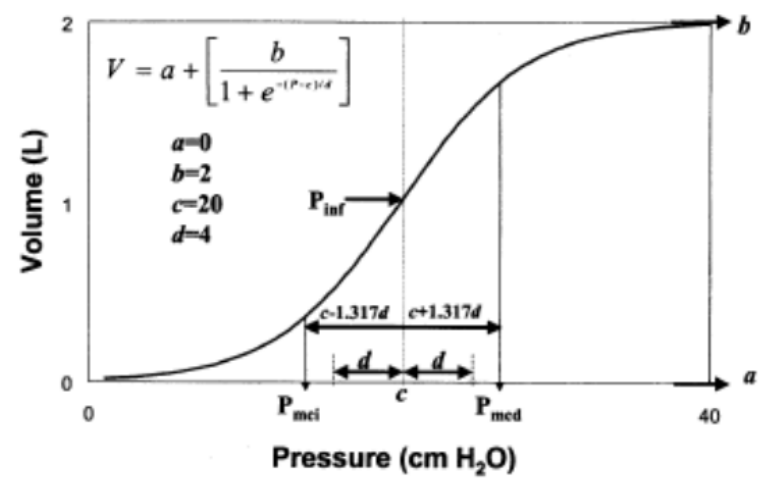

Figure 7: A sigmoidal for curve-fitting pressure-volume data (Harris 2005; Harris, Hess, and Venegas 2000). 


\section{Mesit and Guha}

In our lung simulation, we use this P-V curve to control the volume of lungs that generate the internal pressure force during inhalation and exhalation. In the next section we propose adapting our soft body model using this P-V relation determined from experiment results in Harris (2005) and Harris, Hess, and Venegas (2000) .

The lung model proposed adapts the internal pressure and volume of lung function to our soft body model in Mesit and Guha (2010). We presented the lung as soft body model by using a mass-spring system to control the volume and to generate the elastic behavior of the model. Fluid modeling is used to represent the surface deformation of the model tissue. The internal pressure is used to maintain the volume of the soft body, and gravity is added for realistic physical behavior.

Respiration is a function of the lung's internal pressure force, which controls the lung volume within certain limits. For an appropriately functioning lung model, we adapt this volume control function which depends on internal pressure of the lung. This volume control function specifies the internal pressure inside the lung which leads the air to flow in and out of the lung. The flow of air during inhalation and exhalation is simulated by the number of molecules in the internal pressure model in our simulation.

The internal pressure $\boldsymbol{F}_{\boldsymbol{v}}$, in our lung simulation, is represented as:

$$
\boldsymbol{F}_{v}=\{N, B, C\},
$$

where $N$ is number of molecules, $B$ is the bounding volume of the soft body, and the constraint $C=f(P)$ controls the volume of the lung model.

The constraint, $C$, that controls the volume in our simulation is adopted from the P-V curve control using the sigmoidal equation. The sigmoidal form of a P-V model equation is presented in Venegas, Harris, and Simon (1998) and based on observed performance of both healthy humans, dogs and, humans who have had lung surgery (Narusawa 2001). The P-V data has further been analyzed in acute respiratory distress syndrome in Harris, Hess, and Venegas (2000). We use this sigmoidal equation shown in Figure 7 to generate lung volumes during inhalation and exhalation. The sigmoidal model in P-V curve is presented by:

$$
f(P)=a+\frac{b}{1+e^{-(P-c) / d}},
$$

where $P$ is the respective pressure of the respiratory system, $a$ is the lower asymptote volume, $b$ is the vital capacity, $c$ is the point of maximal compliance (true inflection point), and $d$ is the pressure range that includes most of the volume change.

During inhalation or exhalation, we compute the volume of the lung model by increasing or decreasing the pressure of the respiratory system. The volume function of lung model during inhalation or exhalation is given by:

$$
f(P)=a+\frac{b}{1+e^{-(P t \pm \Delta P-c) / d}},
$$

where $P_{t}$ is the pressure of the respiratory system at the current time and $\Delta P$ is the pressure difference in the current frame and the next frame. $+\Delta P$ and $-\Delta P$ are applied into the volume control function during inhalation and exhalation, respectively. $c=\left\{c_{\text {inf }}, c_{\text {def }}\right\}$ are the points of maximal compliance; $c_{i n f}$ is the point for inhalation and $c_{d e f}$ is the point for exhalation.

The internal pressure force models the inflation and deflation of the lung volume according to $\{+\Delta P$ $\left., c_{\text {inf }}, N_{\text {inf }}\right\}$ and $\left\{-\Delta P, c_{\text {def }}, N_{\text {def }}\right\}$ respectively, where $+\Delta P=40 /$ (number of frames for one respiratory cycle), $c_{\text {inf }}=20, c_{\text {def }}=10, N_{\text {inf }}=70 \mathrm{k}, N_{\text {def }}=100$ in this simulation. The gravitational force is exerted on all surface points of the lung for the real-world physics effects. Finally, all the forces are combined and velocities are evaluated for the new surface positions. 


\section{Mesit and Guha}

We applied the soft body model, where the mass spring system controls the volume of the model, the fluid modeling generates the surface deformation, and the internal pressure produces the inhalation and exhalation activities of lung functions. To maintain the realistic physics, the gravitational force is applied at each surface point. The experiments on body structure control and anamorphosis control from fluidity force show the effects of the soft body parameters. The parameter values, body structure control $(\alpha)$, anamorphosis control $(\beta)$, volume control $(\gamma)$, and gravitation $(\delta)$, are set in Table 2 to present the effect of body structure control from mass-spring system and Table 3 to show the effect of anamorphosis control from fluid modeling.

\subsection{Effect of Body Structure Control on Lung Model}

This experiment observes the dependency of mass-spring on the lung model to control the volume of lung within the limit. The parameter of body structure control $(\alpha)$, is set to $0.25,1$, and 3 while the anamorphosis control $(\beta)$, and volume control $(\gamma)$, are fixed to 1 . For the realism of physics we set the gravitational parameter $(\delta)$ to 0.1 because we need the gravitation force to maintain the center of the lungs. The parameter value sets are shown in Table 2.

Table 2. Parameter values of the effect of body structure control from mass-spring system

\begin{tabular}{|c|c|c|c|c|}
\hline Experiment & $\alpha$ & $\beta$ & $\gamma$ & $\delta$ \\
\hline $\mathbf{A}$ & 0.25 & 1 & 1 & 0.1 \\
\hline $\mathbf{B}$ & 1 & 1 & 1 & 0.1 \\
\hline $\mathbf{C}$ & 3 & 1 & 1 & 0.1 \\
\hline
\end{tabular}

The visualizations of lung simulation during inhalation and exhalation for experiment parameter sets, $\mathrm{A}, \mathrm{B}$, and $\mathrm{C}$ are shown in Figures 8-10 respectively. Each of the Figures 8-10 shows six frames - beginning to the end of inhalation (one to three) and the beginning to the end of exhalation (four to six). Figure 8 shows that with the body structure control set to 0.25 , the lung model at the end of exhalation does not deform back to the volume at the beginning of inhalation. It maintains some volume even though it reaches the end of exhalation and should be in an initial state to start a new cycle of inhalation and exhalation. Figure 9 shows that with the body structure control set to 1.0, the lung model at the end of exhalation deforms back to the original volume better than the result presented in figure 8. However it still maintains some volume at the end of exhalation. Figure 10 illustrates that with body structure control set equal to 3.0, the lung model at the end of exhalation deforms back to the original volume, giving the best result compared to two previous parameter values. The lung volumes of five respiratory cycles in each mass spring parameter are shown in Figure 11. This experiment shows that the body structure control can affect the volume of the lung model thus a good value needs be set to provide the realistic lung simulation. In this experiment, $\alpha=3$ provides a lung volume under control.

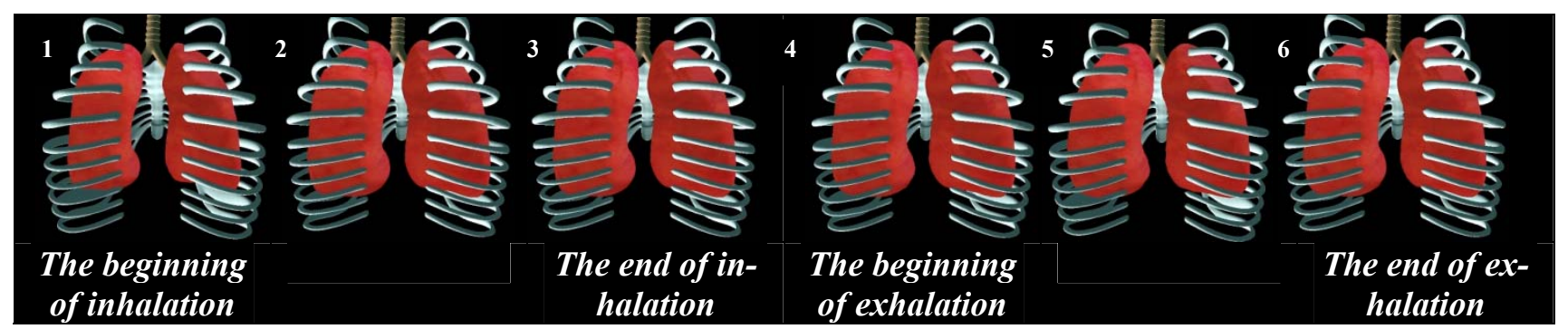

Figure 8: The visual result of lung simulation with parameters of body structure control, anamorphosis control, volume control, and gravitation set to $0.25,1,1$, and 0.1 , respectively. 


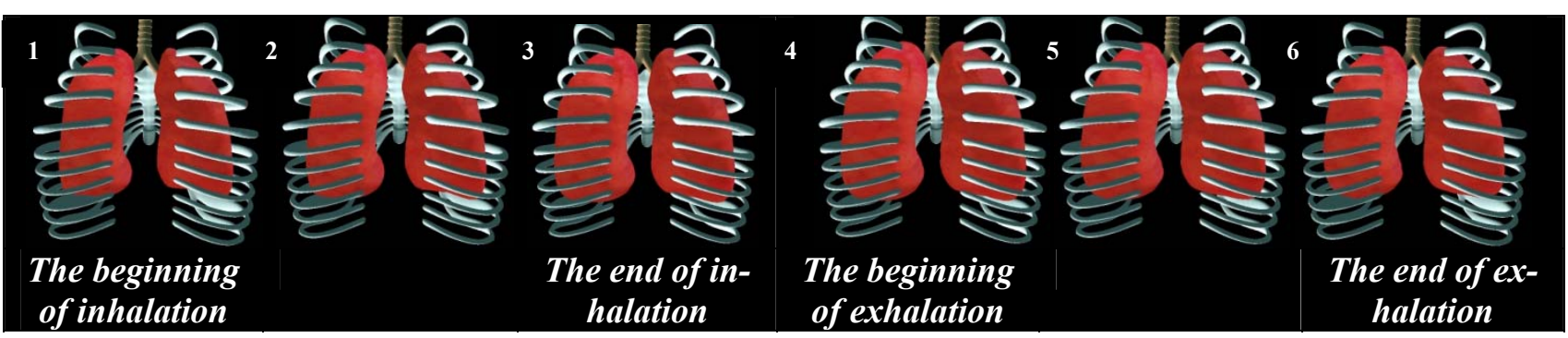

Figure 9: The visual result of lung simulation with parameters of body structure control, anamorphosis control, volume control, and gravitation set to $1,1,1$, and 0.1 , respectively.

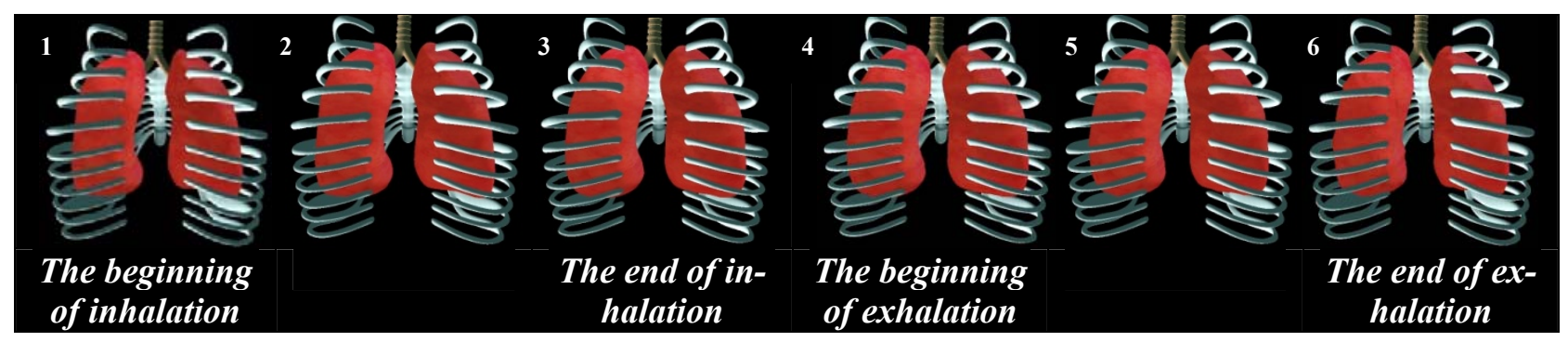

Figure 10: The visual result of lung simulation with parameters of body structure control, anamorphosis control, volume control, and gravitation set to $3,1,1$, and 0.1 , respectively.

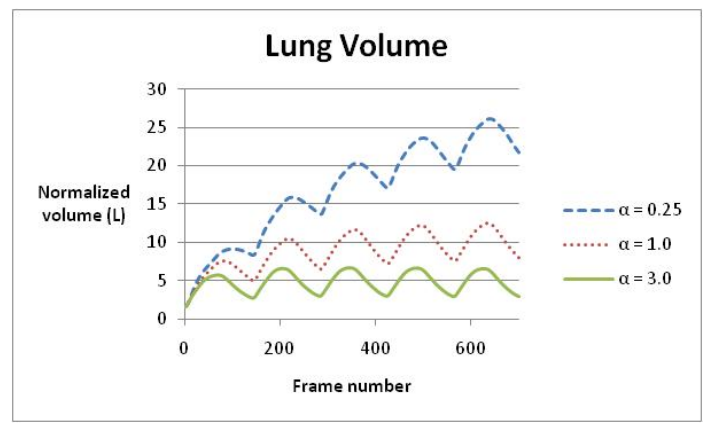

Figure 11: Lung volumes resulted from the experiment parameter sets in Table 2

\subsection{Effect of the Anamorphosis Control from Fluid Force in Lung}

This experiment measures the effect of anamorphosis control from fluid force to create the bumpiness of the lung surface. The parameter of body structure control, $(\alpha)$, and volume control, $(\gamma)$, are fixed to 3 and 1 respectively while the anamorphosis control, $(\beta)$, is varied from $0.25,0.5,0.75,1$, and 2 . Similar to the previous experiment, the gravitational parameter is set to 0.1 . The parameter value sets are shown in Table 3.

Table 3. Parameter values of the effect of anamorphosis control from fluid force.

\begin{tabular}{|c|c|c|c|c|}
\hline Experiment & $\alpha$ & $\beta$ & $\gamma$ & $\delta$ \\
\hline $\mathbf{A}$ & 3 & 0.25 & 1 & 0.1 \\
\hline $\mathbf{B}$ & 3 & 0.5 & 1 & 0.1 \\
\hline $\mathbf{C}$ & 3 & 0.75 & 1 & 0.1 \\
\hline $\mathbf{D}$ & 3 & 1 & 1 & 0.1 \\
\hline $\mathbf{E}$ & 3 & 2 & 1 & 0.1 \\
\hline
\end{tabular}




\section{Mesit and Guha}

The visual results of each anamorphosis parameter value generated by fluidity are shown in Figure 12. Figure 12 is created using a gradient map tool to present the bumpiness of the lung surface. This figure shows that the bumpiness of the surface is increased when the fluid modeling value is increased. Thus, the fluid modeling parameter affects the surface deformation of the lung. This parametric value can be selected to vary with the condition of lung's surface.

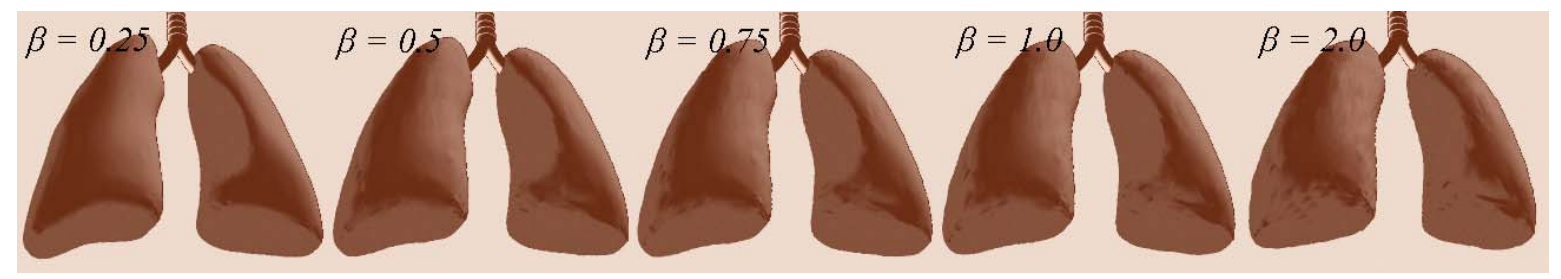

Figure 12: The visual result with gradient map of lung simulation for each anamorphosis parameter value.

\section{CONCLUSION}

We have presented a general formal soft body model with body structure, anamorphosis, and volume controls. For implementation, we selected mass-spring system, SPH fluidity, and internal pressure for those controls respectively. It is also possible to select other methods for these controls. For example, one can use finite element method for all these controls. User defined characteristics such as body structure control, surface anamorphosis control, volume control, constraints, and gravitation can be selected parametrically by users to realistically simulate an intended model. In this general model, body structure control enables the surface deformation while maintaining a relative configuration among surface points, surface anamorphosis helps create realistic deformation on the soft body surface, and volume within the 3D model is maintained by internal pressure. User-defined constraints can influence the amount of deformation, while gravitational force provides natural free fall motion. The simulations of soft ball-like object and lung function show that the algorithm can produce a variety of soft body simulations.

There are several distinct possibilities for future work including, experimenting with different methods for controls, simulation of other internal organs for medical applications, splitting models in real-time for surgical simulations, and further exploration of soft-body models in interactive entertainment, in both standard video game genres or perhaps in an experimental game consisting entirely of soft-body objects.

\section{REFERENCES}

Botsch, M., M. Pauly, M. Gross, and L. Kobbelt. 2006. "PriMo: Coupled Prisms for Intuitive Surface Modeling." In SGP '06: Proceedings of the Fourth Eurographics Symposium on Geometry Processing, 11-20. Aire-la-Ville, Switzerland, Switzerland: Eurographics Association.

Bourg, D.M. 2001. Physics for Game Developers. 1st edition. O'reilly.

Chadwick, J. E., D. R. Haumann, and R. E. Parent. 1989. "Layered Construction for Deformable Animated Characters." In Proceedings of the 16th Annual Conference on Computer graphics and Interactive Techniques - SIGGRAPH '89, 243-252. New York, NY, USA: ACM Press.

Cotes, J. E. 1993. Lung Function Assessment and Application in Medicine. Blackwell Scientific Publication.

Desbrun, M., P. Schröder, and A. Barr. 1999. "Interactive Animation of Structured Deformable Objects." In Proceedings of the 1999 Conference on Graphics Interface '99, 1-8. San Francisco, CA, USA: Morgan Kaufmann Publishers Inc.

Harris, R. S. 2005. "Pressure-Volume Curves of the Respiratory System." RESPIRATORY CARE 50(1):78-98.

Harris, R. S., D. R. Hess, and J. G. Venegas. 2000. "An Objective Analysis of the Pressure-Volume Curve in the Acute Respiratory Distress Syndrome." Am J Respir Crit Care Med 161:432-439. 


\section{Mesit and Guha}

Matyka, M., and M. Ollila. 2003. "Pressure Model of Soft Body Simulation." SIGRAD2003.

Mesit, J., and R. Guha. 2010. "Experimenting with Real Time Simulation Parameters for Fluid Model of Soft Bodies." ANSS2010.

Müller, M., D. Charypar, and M. Gross. 2003. "Particle-based Fluid Simulation for Interactive Applications." In SCA '03: Proceedings of the 2003 ACM SIGGRAPH/Eurographics Symposium on Computer animation, 154-159. Aire-la-Ville, Switzerland, Switzerland: Eurographics Association.

Müller, M., and M. Gross. 2004. "Interactive Virtual Materials." In GI '04: Proceedings of Graphics Interface 2004, 239-246. School of Computer Science, University of Waterloo, Waterloo, Ontario, Canada: Canadian Human-Computer Communications Society.

Müller, M., B. Solenthaler, R. Keiser, and M. Gross. 2005. "Particle-based Fluid-Fluid Interaction." In SCA '05: Proceedings of the 2005 ACM SIGGRAPH/Eurographics Symposium on Computer Animation, 237-244. New York, NY, USA: ACM. doi: http://doi.acm.org/10.1145/1073368.1073402.

Narusawa, U. 2001. "General Characteristics of the Sigmoidal Model Equation Representing Quasi-Static Pulmonary P-V Curves." J Appl Physiol 91(1):201-210.

Palsson, B., J. A. Hubbell, R. Plonsey, and J. D. Bronzino. 2003. Tissue Engineering (Principles and Applications in Engineering). CRC Press.

Santhanam, A. P. 2003. "Modeling, Simulation, and Visualization of Three-Dimensional Lung Dynamics." Ph.D. Thesis, University of Central Florida. Orlando, FL, USA.

Sederberg, T. W., and S. R. Parry. 1986. "Free-form Deformation of Solid Geometric Models." $A C M$ SIGGRAPH Computer Graphics 20(4):151-160. doi: 10.1145/15886.15903.

Stoiber, N., R. Seguier, and G. Breton. 2010. "Facial Animation Retargeting and Control Based on a Human Appearance Space." Computer Animation and Virtual Worlds 21(1):39-54. doi: 10.1002/cav.331.

Venegas, J. G., S. Harris, and B. A. Simon. 1998. “A Comprehensive Equation for the Pulmonary Pressure-Volume Curve.” J Apply Physiol 84:389-395.

\section{AUTHOR BIOGRAPHIES}

JARUWAN MESIT is a visiting professor in the Department of Computer Science at Grambling State University. She received her B.Sc. and M.S. in computer science from Rajabhat Institute Phetchaburi and National Institute of Development Administration, Thailand and received her Ph.D. at University of Central Florida, USA in 2010. Her research interest is in the area of computer simulations in physics-based modeling, visualizing soft bodies, and collision detection among soft bodies and soft bodies-solid objects. Her e-mail address is jmesit@cs.ucf.edu.

RATAN GUHA is a professor in the Department of Electrical Engineering and Computer Science at the University of Central Florida. He received his B.Sc. degree with honors in Mathematics and M.Sc. degree in Applied Mathematics from University of Calcutta and received the Ph.D. degree in Computer Science from the University of Texas at Austin in 1970. He has authored over 150 papers published in various computer journals, book chapters and conference proceedings. His research has been supported by grants from ARO, NSF, STRICOM, PM-TRADE, NASA, and the State of Florida. He has served as a member of the program committee of several conferences, as the general chair of CSMA'98 and CSMA'2000 and as the guest co-editor of a special issue of the Journal of Simulation Practice and Theo$r y$. He is a member of ACM, IEEE, and SCS and served as a member of the Board of Directors of SCS from 2004 to 2006. He is currently serving in the editorial board of Modeling and Simulation in Engineering published by Hindawi Publishing Corporation. His e-mail address is guha@cs.ucf.edu. 